\title{
Disfunção cognitiva em doença renal crônica pré-dialítica: uma revisão sistemática
}

\author{
Cognitive dysfunction in pre-dialysis chronic kidney disease: \\ a systematic review
}

\section{Disfunción cognitiva en la enfermedad renal crónica previa a la diálisis: una revisión sistemática}

\author{
Leopoldo Antônio Pires ${ }^{1}$, Rogério Baumgratz de Paula ${ }^{2}$, Danielle \\ Guedes de Andrade Ezequiel ${ }^{3}$, Natália Maria da Silva Fernandes ${ }^{4}$
}

1.Médico Neurologista, Professor de Neurologia e Doutorando do Programa de Pós Graduação em Saúde da Universidade Federal de Juiz de Fora, Juiz de Fora-MG, Brasil. ORCID 0000-0002-8636-3931

2.Médico Nefrologista, Professor Titular de Nefrologia na Universidade Federal de Juiz de Fora e Doutor em Nefrologia pela Universidade Federal de São Paulo, São Paulo-SP, Brasil. ORCID 0000-0002-77782907

3. Médica Endocrinologista, Professora Adjunta de Endocrinologia e Doutora em Saúde, Universidade Federal de Juiz de Fora, Juiz de Fora-MG, Brasil. ORCID 0000-0002-2120-2838

4. Médica Nefrologista, Professora Associada de Nefrologia e Doutora em Saúde, Universidade Federal de Juiz de Fora, Juiz de Fora-MG, Brasil. ORCID 0000-0001-8728-7937

\begin{abstract}
Resumo
Introdução. No Brasil, a população com mais de 65 anos pode chegar a $25,5 \%$, em 2060. Com o envelhecimento populacional ocorre um aum ento da prevalência das doenças degenerativas, dentre as quais o comprometimento cognitivo (CC). Impacto semelhante é observado na doença renal crônica (DRC). Existem fatores fisiopatológicos comuns entre a DRC e o CC. Dentre pacientes com DRC, até 38\% apresentam CC. Em relação à fisiopatologia das alterações cognitivas na DRC, os achados são controversos. Objetivo. Avaliar os estudos relacionados à disfunção cognitiva na população com DRC pré-dialítica. Método. Utilizamos as estratégias PRISMA. Selecionamos os unitermos "renal insufficiency, chronic" and "cognitive disfunction", realizamos a revisão sistemática e não meta-análise dada a disparidade dos estudos. Utilizamos as bases de dados disponíveis de forma gratuita: PubMed, Scielo. A seleção dos artigos foi realizada por dois pesquisadores. Critérios de inclusão: apresentar resumo em língua portuguesa, inglesa, espanhola ou francesa; incluir pacientes em pré-diálise; utilizar avaliações clínicas e/ou laboratoriais e/ou de imagens. Resultados. Encontramos 116 artigos e avaliamos 51 . Conclusão. O CC é frequente na DRC e a etiologia permanece em discussão. A maioria dos estudos é derivada de dados secundários de grandes coortes ou estudos transversais. Os instrum entos clínicos mais utilizados são o MEEM e o MoCA e de neuroimagem, a ressonância magnética. Poucos estudos avaliam a inflamação e as toxinas urêmicas. Os mecanismos do CC na DRC são diversos, nem sempre correlacionados, existindo uma grande lacuna no melhor entendimento da associação fisiopatológica destas duas importantes morbidades.
\end{abstract}

Unitermos. Disfunção Cognitiva; demência; doença renal; insuficiência renal crônica

\footnotetext{
Abstract

Introduction. In Brazil, the population over 65 years old can reach $25.5 \%$, in 2060 . With population aging, there is an increase in the prevalence of degenerative diseases, including cognitive im pairment (CI). A similar im pact is seen in chronic kidney disease (CKD). There are pathophysiological factors common between CKD and CI. Among CKD patients, up to 38\% have CI. Regarding the pathophysiology of cognitive changes in CKD, the findings are controversial. Objective. Is to evaluate studies related to cognitive dysfunction in the population with pre-dialysis CKD. Method. We use PRISMA strategies. We selected the
} 
keywords "renal insufficiency, chronic" and "cognitive disfunction", carried out a systematic review and not meta-analysis given the disparity in the studies. We use the databases available free of charge: PubMed, Scielo. The selection of articles was carried out by two researchers. Inclusion criteria: present an abstract in Portuguese, English, Spanish or French; include predialysis patients; use clinical and / or laboratory evaluations and / or images. Results. We found 116 articles and evaluated 51. Conclusion. CI is common in CKD and the etiology remains under discussion. Most studies are derived from secondary data from large cohorts or cross-sectional studies. The most used clinical instruments are MMSE and MoCA and neuroimaging, magnetic resonance. Few studies have evaluated inflammation and uremic toxins. The mechanisms of CI in CKD are diverse, not always correlated, and there is a big gap in the better understanding of the pathophysiological association of these two important morbidities.

Keywords. Cognitive dysfunction; dementia; kidney disease; chronic kidney insufficiency

\section{RESUMEN}

Introducción. En Brasil, la población mayor de 65 años puede llegar al 25,5\%, en 2060. Con el envejecimiento de la población, aumenta la prevalencia de enfermedades degenerativas, incluido el deterioro cognitivo (CC). Se observa un impacto similar en la enfermedad renal crónica (ERC). Existen factores fisiopatológicos comunes entre ERC y CC. Entre los pacientes con ERC, hasta un 38\% tienen CC. En cuanto a la fisiopatología de los cambios cognitivos en la ERC, los hallazgos son controvertidos. Objetivo. Es evaluar los estudios relacionados con la disfunción cognitiva en la población con ERC prediálisis. Método. Utilizamos estrategias PRISMA. Se seleccionaron las palabras clave "insuficiencia renal crónica" y "disfunción cognitiva", se realizó una revisión sistemática y no un metaanálisis dada la disparidad de los estudios. Utilizamos las bases de datos disponibles de forma gratuita: PubMed, Scielo. La selección de artículos estuvo a cargo de dos investigadores. Criterios de inclusión: presentar un resumen en portugués, inglés, español o francés; incluir pacientes en prediálisis; utilizar evaluaciones y / o imágenes clínicas y / o de laboratorio. Resultados. Encontramos 116 artículos y evaluamos 51. Conclusión. La CC es común en la ERC y la etiología permanece en discusión. La mayoría de los estudios se derivan de datos secundarios de grandes cohortes o estudios transversales. Los instrumentos clínicos más utilizados son MMSE y MoCA y neuroimagen, resonancia magnética. Pocos estudios han evaluado la inflam ación y las toxinas urémicas. Los mecanismos de CC en la ERC son diversos, no siempre correlacionados, y existe una gran brecha en la mejor comprensión de la asociación fisiopatológica de estas dos importantes morbilidades.

Palabras clave. Disfunción cognitiva; demencia; nefropatia; insuficiencia renal crónica

Trabalho realizado na Universidade Federal de Juiz de Fora, Juiz de Fora-MG, Brasil.

Endereço para correspondência: Natália MS Fernandes. R. Jamil Altaf 132. Bairro Vale do Ipê. Juiz de Fora-MG, Brasil. CEP 36035-380. Email: nataliafernandes02@gmail.com

\section{INTRODUÇÃO}

O conceito de cognição é abrangente e complexo e definido como ato ou processo de aquisição de conhecimento que se dá por meio de percepção, atenção, associação, memória, raciocínio, juízo, imaginação, pensamento e linguagem ${ }^{1}$. Ou seja, seria o "conjunto de processos mentais utilizados no pensamento, na classificação, no 
reconhecimento e na compreensão, para o julgamento por meio do raciocínio, para o aprendizado de determinados sistemas e para a solução de problemas". Cabe ressaltar que, além do aspecto individual, existe o aspecto social por ser a cognição um processo pelo qual o ser humano interage com os seus semelhantes e com o meio em que vive, sem perder a sua identidade existencial. Trata-se, portanto, de um processo de conhecimento, que tem como substrato a informação obtida através da interação com o meio. Seu pleno funcionamento depende da integridade anatômica e funcional do sistema nervoso e o que se encontra registrado por meio da percepção, da atenção e da possível associação com comprometimento secundário da memória, do raciocínio, do juízo, da imaginação, do pensamento e da linguagem ${ }^{1}$.

Quando ocorre algum distúrbio neste processamento, utiliza-se o termo comprometimento cognitivo ou déficit cognitivo (DC) que levará a diversos prejuízos sejam individuais ou sociais. O DC pode ocorrer em qualquer fase da vida, existindo dois momentos de maior risco: o primeiro, durante $o$ desenvolvimento embrionário que inclui malformações, infecções, ação de drogas e medicações, dentre outras ${ }^{2-4}$ e o segundo, durante o processo de envelhecimento, quando ocorre aumento da incidência das doenças degenerativas tais como hipertensão arterial sistêmica (HAS), diabetes mellitus (DM), além dos quadros demenciais, como a doença de Alzheimer (DA). Merece destaque, a ocorrência de comprometimento cognitivo sem 
repercussão funcional quadro este denominado de comprometimento cognitivo leve (CCL), condição clínica frequentemente subdiagnosticada. O CCL manifesta-se por declínio em um ou mais domínios cognitivos, sem que haja comprometimento funcional, característica obrigatória para o diagnóstico de demência. Tal condição apresenta diversas etiologias, incluindo doenças clínicas principalmente HAS e DM, polifarmácia, depressão, dentre outras. Na maioria das vezes, corresponde a um estágio ainda incipiente de demência, como ocorre na doença de Alzheimer e nas demências vasculares ${ }^{5-7}$.

De acordo com a Organização Mundial da Saúde (OMS) e a Organização das Nações Unidas (ONU) tem se observado aumento da expectativa de vida, em nível mundial. Em 2050, espera-se que a população mundial com idade $\geq 60$ anos chegue a dois bilhões, em contraponto aos 900 milhões observados em $2015^{8}$. Atualmente, 125 milhões de pessoas têm 80 anos ou mais e em 2050, as previsões apontam que existirão 434 milhões de pessoas nesta faixa etária, em todo o mundo. Além disso, previsões apontam que em 2050, 80\% dos indivíduos idosos viverão em países de baixa e média renda com consequente aumento da demanda para os sistemas de saúde ${ }^{9}$. No Brasil, segundo o IBGE, a parcela da população com mais de 65 anos, em 2018, era de 10,5\%, podendo chegar a 15\% em 2034 e 25,5\%, em 2060. Com o envelhecimento populacional ocorre um aumento da prevalência das doenças degenerativas, dentre as quais as demências, cuja etiologia mais prevalente é a DA, 
diagnosticada em 55 a $60 \%$ dos $\operatorname{casos}^{10-12}$, seguida pela demência vascular (DV), presente em 15 a 20\% ${ }^{5,13}$.

Do ponto de vista epidemiológico, impacto semelhante é observado na doença renal crônica (DRC), que representa a quinta causa de anos perdidos de vida ${ }^{14,15}$ e que tem como fatores etiológicos principais a HAS e o DM, cuja prevalência tem assumido elevadas taxas, em nível mundial7,16. Ademais existem fatores fisiopatológicos comuns entre a DRC e as demências, como o envelhecimento, a raça não branca, 0 baixo nível socioeconômico, a baixa escolaridade e a dislipidemia, associados a elementos específicos decorrentes da DRC, como anemia, albuminúria, retenção de solutos urêmicos, inflamação, estresse oxidativo, calcificações vasculares, hiponatremia e fatores ligados à diálise ${ }^{17}$.

A DRC evolui de forma insidiosa e progressiva, do estágio 1 ao estágio $5^{18}$, sendo considerada fator de risco independente para $\mathrm{DC}^{19}$. Nos estágios iniciais, ainda é possível retardar a progressão da DRC, porém no estágio 5, o paciente poderá necessitar de terapia renal substitutiva (TRS), seja dialítica ou transplante renal. Dentre estes pacientes com DRC estágios avançados, 16 a $38 \%$ apresentam comprometimento cognitivo, incluindo $\mathrm{CCL}$ e demência, sendo o diagnóstico destas alterações de grande relevância no processo de avaliação clínica. A demência se associa com maior risco de morte, menor adesão ao tratamento, suspensão da diálise, hospitalização e aumento no custo do atendimento. Além disso, dificulta a aderência ao complexo plano terapêutico necessário para os cuidados 
destes indivíduos, que inclui medidas não farmacológicas, como dieta e atividade física, além da prescrição de múltiplas medicações ${ }^{20}$. Desta forma, o diagnóstico precoce e abordagem objetiva do DC podem melhorar os cuidados clínicos na $\mathrm{DRC}^{17}$, proporcionando um período de "janela terapêutica" para possíveis intervenções nos fatores de risco modificáveis e possíveis complicações relacionadas ${ }^{19}$.

Em relação à fisiopatologia das alterações cognitivas na DRC, os achados são controversos. Ademais existem poucos trabalhos que avaliam as alterações cognitivas em estágios iniciais da DRC. Alguns estudos atribuem tais alterações à presença de lesões vasculares encefálicas, principalmente da substância branca, enquanto outros sugerem a presença de alterações cognitivas, mesmo na ausência de lesões à ressonância magnética (RM) do encéfalo. Neste contexto, o estudo e o conhecimento sobre as especificidades das alterações cognitivas associadas à DRC poderiam contribuir para precocidade nas intervenções e orientações, melhorando o tratamento e a qualidade de vida do paciente com DRC e de seus cuidadores.

O objetivo desse trabalho é avaliar os estudos relacionados à disfunção cognitiva na população com doença renal crônica pré-dialítica.

\section{MÉTODO}

Utilizamos as estratégias PRISMA (Principais Itens para Relatar Revisões sistemáticas e Meta-análises), passíveis de serem utilizadas no nosso estudo. Selecionamos os 
unitermos "renal insufficiency, chronic" and "cognitive disfunction", obedecendo aos critérios PICO (Participantes, Intervenções, Comparações e Resultado), porém salientando não estarmos avaliando intervenções ou desfechos e sim a associação entre os dois termos. Também decidimos realizar apenas a revisão e não meta-análise dada a disparidade dos estudos, pois nosso interesse foi de avaliar as associações e os métodos utilizados para encontrá-las. Demonstrando que neste campo, ainda não temos consenso sobre tais abordagens. Utilizamos as bases de dados disponíveis de forma gratuita - PubMed, Scielo.

Dito isto, observamos inicialmente que os artigos mais específicos começam a aparecer a partir de 2012, até então o termo "cognitive dysfunction" não constava nos termos Mesh, havia apenas o termo "cognition" que ampliava muito os horizontes, tornando a busca muito inespecífica.

A seleção dos artigos foi realizada por dois pesquisadores utilizando um formulário padrão (LAP,NMSF). A seleção foi então confrontada e os artigos selecionados após consenso entre os pesquisadores.

Os critérios de inclusão foram: apresentar resumo em língua portuguesa, inglesa, espanhola ou francesa; incluir pacientes em pré-diálise em quaisquer estágios; utilizar avaliações clínicas e/ou laboratoriais e/ou de imagens; revisões de abordagens neurocognitivas também foram aceitas. Como critérios de não inclusão: apresentar resumos em outras línguas que não as citadas acima, incluir apenas 
pacientes em terapia renal substitutiva ou incluir pacientes com insuficiência renal aguda.

\section{RESULTADOS}

No dia 09 de maio de 2019, utilizando os termos Mesh no Medline, "renal insuficiency, chronic" and "cognitive disfunction" encontramos 77 artigos e destes foram selecionados 37 que preenchiam os critérios do escopo da pesquisa.

No dia 27 de janeiro de 2020, utilizando os mesmos unitermos, realizamos uma atualização da pesquisa e encontramos 106 artigos, 36 novos, destes selecionamos 11.

Além disto, acrescentamos três estudos com dados relevantes sobre imagem e metabolismo cerebral, pertinentes a revisão, não encontrados com os unitermos. Ao final, a partir dos unitermos, analisamos 48 artigos e acrescentamos outros três, totalizando 51 (Figura 1).

\section{DISCUSSÃO}

Com finalidade didática agruparemos os achados dos estudos em tópicos para discussão dos mesmos, excluídas as revisões que estão incluídas em vários tópicos. Inicialmente as manifestações neurológicas na DRC eram atribuídas a encefalopatia urêmica e suas complicações ${ }^{21-24}$. 
Figura 1. Fluxograma da Revisão.

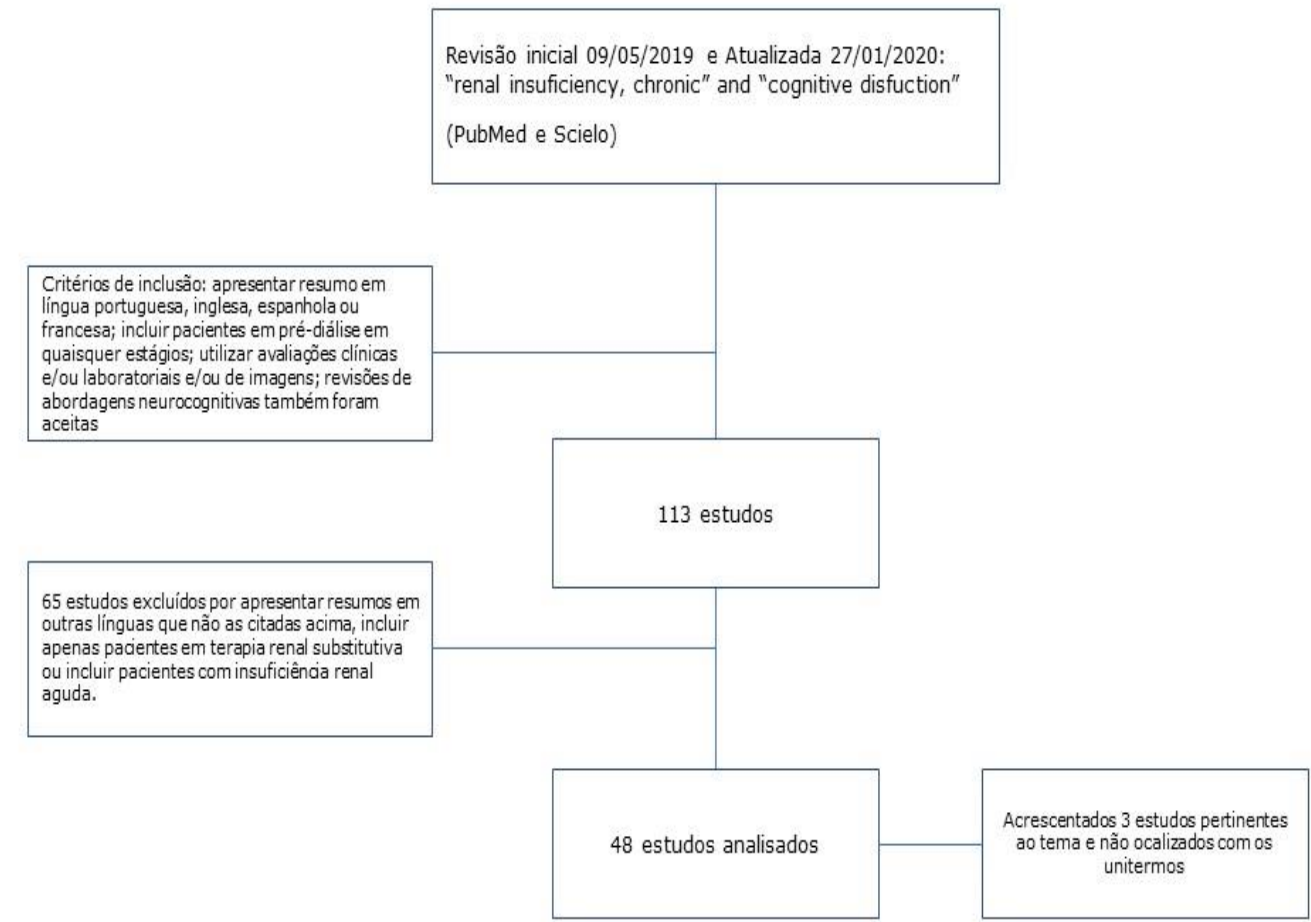

Posteriormente surgiram relatos da ocorrência das demências, sendo estas atribuídas principalmente a alterações vasculares que ocorrem nas fases avançadas da $D_{R C}{ }^{25}$ ou secundária à diálise ${ }^{26}$. A associação entre alterações cognitivas e DRC começaram a serem encontradas em estudos a partir dos anos 2000. Isto se deve, provavelmente, ao fato do termo "cognitive disfuction" ter sido colocado como unitermo no Medline a partir de 2012. Anteriormente era utilizado o termo "cognition", porém este era muito amplo diminuindo a especificidade da pesquisa.

É interessante notar que $41 \%$ dos estudos são asiáticos (Japão 27-33, China ${ }^{34-39}$, Taiwan ${ }^{40-44}$ e India ${ }^{45,46}$ ), 25\% realizados nos EUA ${ }^{17,47-56}$ e $20 \%$ são europeus (Itália ${ }^{57-59}$, 
Holanda ${ }^{60,61}$, Alemanha ${ }^{62,63}$, França ${ }^{64}$ e Grécia ${ }^{65}$ ). No Brasil temos $8 \%$ dos estudos ${ }^{66-70}$. Com menor frequência, $2 \%$ destes no Canadá71, $2 \%$ em Israel $^{72}$ e $2 \%$ no México ${ }^{73}$. (Figura 2).

Figura 2. Localização geográfica dos estudos.

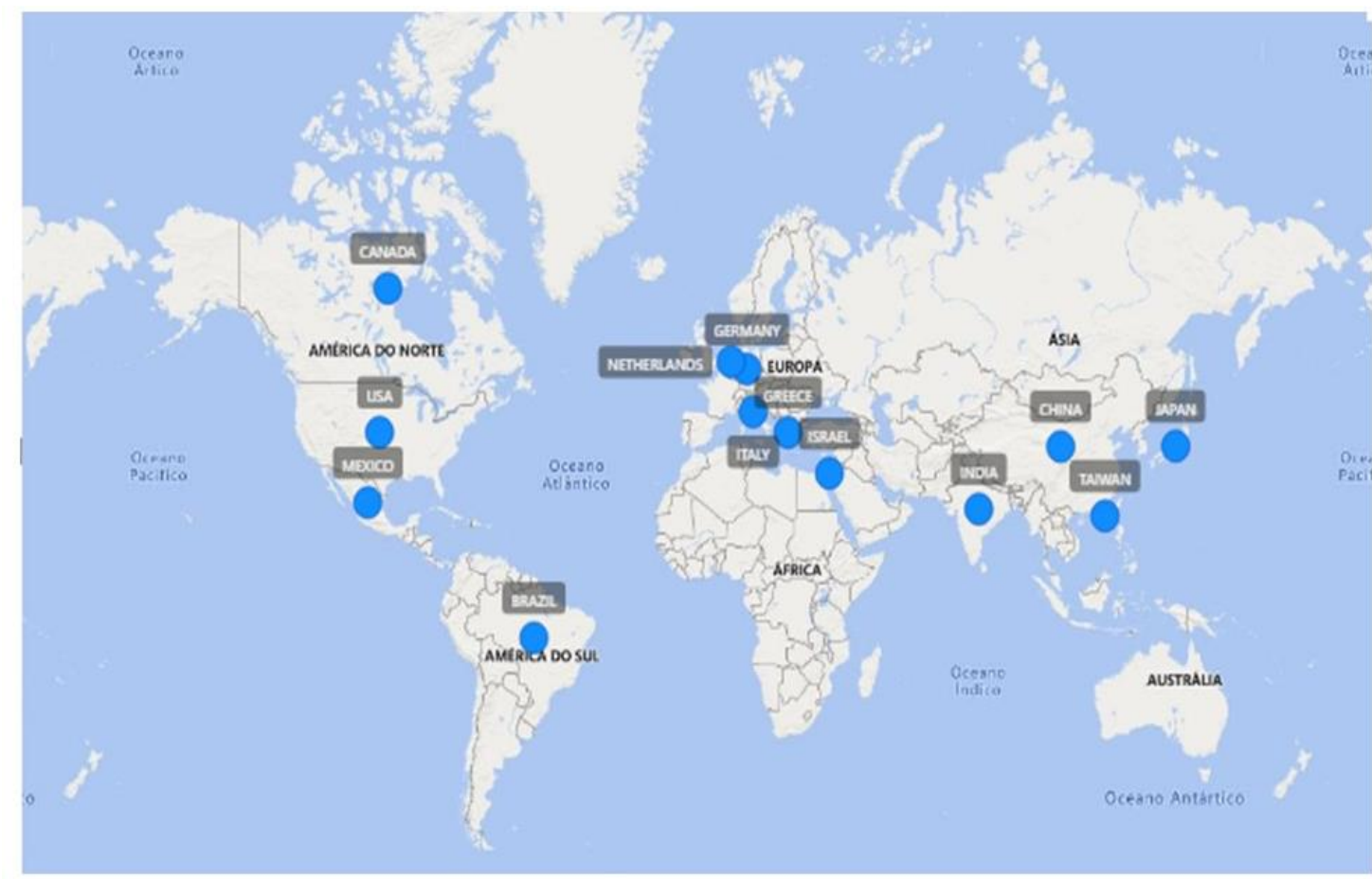

Relacionado ao desenho do estudo chama a atenção o grande número de revisões $(23 \%)^{29,30,36,48,49,52,54,60,68,69}$ comparado ao número de estudos originais. Os estudos transversais são maioria $(40 \%)^{32-34,37,39,41,42,44,51,53,55,57-}$ $59,63,65,66$, seguidos pelo de coorte, vale ressaltar que dentre estes, vários são aninhados, ou seja, há uma grande coorte "guarda-chuva" e nela são incluídas diversas perguntas

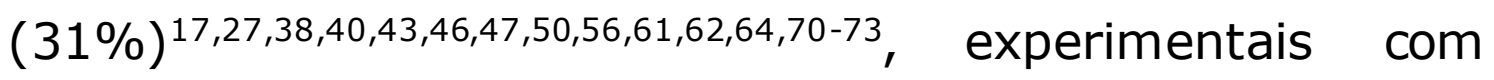
$4 \%{ }^{31,35}$ e caso-controle $2 \%{ }^{45}$. Em um único caso o estudo foi tanto transversal como longitudinal ${ }^{28}$ (Gráfico 1 ). 


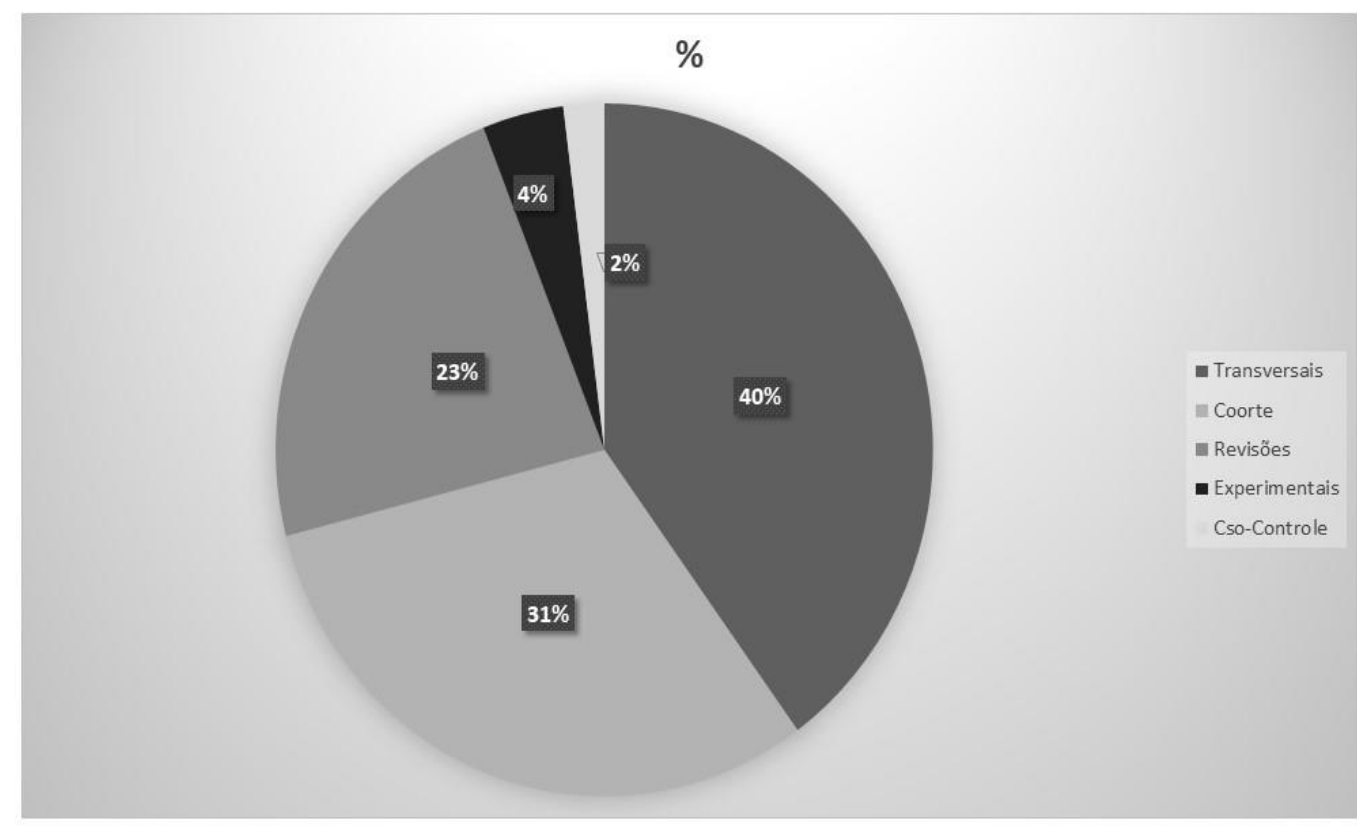

Excluindo os artigos de revisão, nos quais não houve amostragem populacional, a amostragem das populações estudadas foram, em sua maioria, amostras por conveniência (70\%). Do total, 5,4\% dos estudos foram experimentais e estes utilizam metodologia diversa para cálculo de amostra. Vale ressaltar que os estudos que apresentavam algum tipo de grupo controle não foram $(21,6 \%)$ estudos do tipo caso-controle no qual se definiu o tamanho e da amostra e o poder do estudo previamente. Apenas um estudo foi definido como caso-controle ${ }^{45}$, porém, mesmo neste caso, não houve cálculo amostral ou de poder do estudo. Isto não diminui o valor dos achados deles, pois, são majoritariamente, estudos de vida real quer de coorte quer transversais. Estes estudos têm como principal objetivo demonstrar os achados clínicos nesta população de pacientes 
com DRC e alterações cognitivas, são estudos exploratórios, para levantar perguntas específicas, como por exemplo: as alterações são funcionais? Ou despertar para estudos de intervenção, tais como: diminuição dos níveis de toxina urêmica melhora a cognição? Diminuição ou alteração do padrão das interleucinas pró e anti-inflamatórias alteram a cognição? (Gráfico 2).

Gráfico 2. Tipos de amostra.

Conveniência

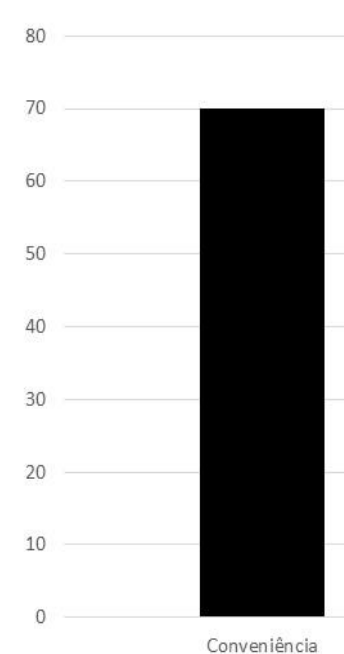

(n)

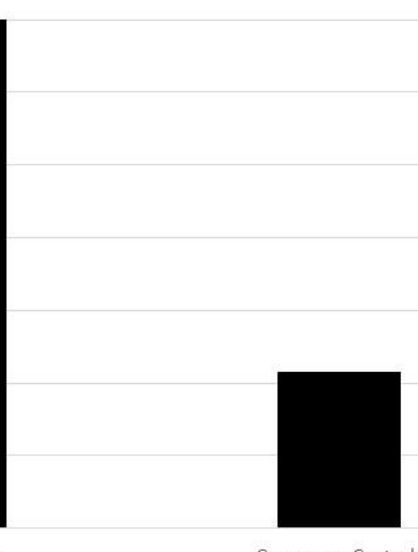

- $\%$

O tamanho da amostra variou, principalmente nos estudos de coorte de grande duração, o maior deles, um estudo em uma coorte brasileira de 15.505 indivíduos ${ }^{67}$ seguido pelo estudo dos EUA ${ }^{17}$ com 3883 pacientes, acompanhados por seis anos até estudos que ao início havia 52 pacientes e ao final do estudo $40^{64}$. Avaliando apenas estudos realizados em humanos e excluindo revisão de artigos, observamos que 57\% destes tinham mais de 200

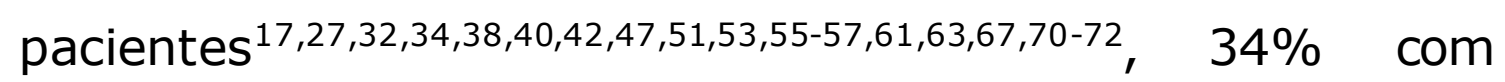


amostra menor que $100^{39,41,43,45,46,50,58,62,64,66}$, e $9 \%$ entre 100 e 200 pacientes $33,37,44,59,65$.

Em apenas sete estudos tivemos grupo controle $41,42,46,47,55,58,62$. Em revisões costuma-se somar o número de pacientes incluídos nos estudos avaliados porém, diante da heterogeneidade dos mesmos, esta informação, nesta revisão, é mais ilustrativa e foi composta de mais de 32.007 pacientes.

Com relação aos testes clínicos utilizados para avaliação cognitiva, estes foram realizados de forma heterogênea. Na ampla maioria dos casos a cognição global foi avaliada pelo Mini Exame do Estado Mental (MEEM) 74,75 e posteriormente pela sua versão modificada, o Mini Exame do Estado Mental Modificado (Modified Mini State Exam - 3MS) ${ }^{76,77}$, seguido pelo Montreal Cognitive Assessment - MoCA ${ }^{78}$. Os domínios específicos foram abordados por uma diversidade de testes com destaque para o Teste de Trilhas $B$, Fluência verbal (categoria animais) e para as funções executivas.

O MEEM foi utilizado em 27 estudos, porém em 4 ocasiões foi utilizada a versão modificada (Modified MMSE 3MS) isolado ou em conjunto. O Moca foi utilizado em cinco estudos ${ }^{33,64,66,71,72}$ e citado em duas revisões ${ }^{48,52}$.

Com relação a avaliação das alterações na concentração sanguínea das toxinas urêmicas na DRC, assim como da inflamação que ocorre neste grupo de pacientes, observamos algumas avaliações. Os exames laboratoriais foram realizados na maioria dos estudos, sendo o uso da taxa de filtração glomerular estimada (TFGe) e a 
classificação da DRC em estágios o padrão adotado. Dosagem de alguns fatores inflamatórios, PTH, Vit D, homocisteína entre outros, além da avaliação da relação proteinúria/albuminúria foram realizados na maioria deles. Observamos[/NF1] estudos que avaliaram os fatores inflamatórios ${ }^{39,64,65}$, a correlação de anemia com o DC28,46.

Em um único estudo ${ }^{64}$ foram realizadas as dosagens das toxinas urêmicas (Ácido Indol acético, PCresil sulfato e Indoxil sulfato) e em outro estudo se correlacionou a dosagem da Vit. D com DRC e $D^{36}$ e em um terceiro encontraram que níveis elevados de homocisteina correlacionaram-se com comprometimento da função executiva e não com da memória ${ }^{47}$. Sugerindo a coexistência da hipótese vascular e não vascular associadas a disfunção cognitiva na DRC.

Com o advento da Tomografia computadorizada em 1972, a Ressonância Magnética em 1973/1976, e, a partir de 1992, a RM funcional (fRM) abriram-se novas formas de avaliação da anatomia e função cerebrais ${ }^{79,80}$. Estes métodos de neuroimagem fornecem meios não invasivos representando uma abordagem importante para elucidar ainda mais a conexão rim-cérebro em indivíduos com DRC e se correlacionam com a presença de atrofia cerebral e alterações na densidade cerebral em pacientes com DRC; doença vascular cerebral, incluindo hiperintensidade e lesões da substância branca, microsangramentos cerebrais, infartos cerebrais silenciosos e infartos corticais, em pacientes com DRC; e nas semelhanças no fluxo sanguíneo cerebral 
regional entre pacientes com DRC e pacientes com distúrbios afetivos $^{81}$.

Nesta revisão em 17 estudos citaram a RM, sendo que em sete destes, utilizou-se o aparelho de 3 teslas e, em seis, aparelhos de 1,5 teslas e em quatro, foi citado como instrumento de avaliação 29,30,49,52. A Tomografia por emissão de pósitron (Pet Scan) foi citada apenas em uma revisão ${ }^{29} \mathrm{e}$ na composição de grupo com CCL relacionado a doença de Alzheimer em um estudo ${ }^{59}$.

O EEG, um exame que avalia a função eletrofisiológica sendo assim algumas alterações podem contribuir para análise de função cognitiva. Contudo, trata-se de um método não rotineiramente utilizado com este propósito. Na nossa revisão foi utilizado em dois estudos ${ }^{43,59}$.

Exceto por dois estudos que não encontraram associação entre DC e DRC 34,63 a maioria dos estudos refere aumento da prevalência de DC associada a DRC. Estudo realizado em uma coorte brasileira de 15.505 indivíduos sugere que o desempenho cognitivo permanece preservado até a função renal atingir piora significativa e que medidas preventivas da progressão da DRC podem contribuir para a preservação da função cognitiva. Porém, trata-se de uma análise secundária do ELSA Brasil, não desenhado especificamente para avaliar $\mathrm{DC}^{67}$. 
Quadro 1. Sumarizando as associações entre doença renal crônica e disfunção cognitiva.

1- Como analisar a clinicamente a cognição na DRC?

- MoCA*

- MEEM e 3Ms\#

- Testes de função executiva (Trilhas B, Fluência verbal (categoria animais) $\mathbb{R}$

2- Quais os exames de imagem disponíveis/utilizados?

- Tomografia computadorizada\#

-Ressonância Magnética (RM)* / RM com espectroscopia ${ }^{\circledR}$

- Tomografia com emissão de pósitrons Scan $®$

-Eletroencefalograma ${ }^{\circledR}$

3- Alterações analisadas na Doença Renal Crônica que podem influenciar a função cognitiva:

- Anemia (por déficit de eritropoetina e ferro)\#

- Vitamina D, PTH\#

- Toxinas urêmicas (Ac. Indol acético, PCresil sulfato e Indoxil sulfato) ${ }^{\circledR}$

- Inflamação (PCRus, IL6, IL10, TGFß\#; Outros: IL 35, IL23®)

- Padrão de resposta imune (Treg/TH17) $®$

- Beta amilóide, pTau $181 \circledR$

- Grelina $®$

4- Como tratar? (c)

- Retardar a queda da TFG?

- Melhor controle DM e HAS?

- EPO?

- BRA?

- Terapia Renal Substitutiva - trata ou associa fatores de progressão do DC?

* Mais indicado; \#frequentemente avaliados; ®Raramente avaliados; @Sem consenso. MoCA- Montreal Cognitive Assessment; MEEM- Mini Exame do Estado Mental; 3Ms-;PTH- paratohormônio; PCRus- proteína $C$ reativa ultrassensível;IL- interleucina; Treg- resposta imunológica T reguladora; resposta imunológica Th17-; pTau- proteína Tau;TFG- taxa de filtração glomerular; DM- diabetes mellitus; HAS- hipertensão arterial sistêmica; EPO- eritropoetina; BRA- bloqueador de receptor de angiotensina; DC- disfunção cognitiva. 
A avaliação cognitiva foi realizada utilizando testes de avaliação da cognição global como Mini Exame do Estado Mental (MEEM), O Mini Exame do Estado Mental Modificado (3MS) e o Montral Cognitive Assessment (MoCA). A baixa sensibilidade do MEEM na deteç̧ão do CCL é uma grande crítica ao uso desta ferramenta nas avaliações cognitivas. $O$ 3MS reduziu este déficit e o MoCA apresenta maior sensibilidade na precocidade desta condição ${ }^{66}$. Com relação aos domínios afetados, observaram-se principalmente alterações na velocidade de processamento e disfunções executivas $29,32,42,64,68$. As ferramentas mais utilizadas para detectar esta condição foram o Teste de Trilhas $B$, a fluência verbal semântica e fonêmica e o Digit Span.

Não foram encontradas referências as comorbidades frequentemente encontradas na população com DRC e DC. É fato que depressão está associada a DC e que o tratamento desta pode melhorar a função cognitiva ${ }^{58,64}$. Outras comorbidades associadas a fatores de risco cardiovasculares clássicos, principalmente HAS e DM, estão entre as principais causas de DRC e associadas ao aumento significativo da prevalência de DC de natureza vascular ${ }^{65}$.

Uma complicação frequentemente encontrada na DRC é a anemia, secundária a carência de ferro e a déficit de produção de eritropoietina. Em indivíduos com DRC, a presença de anemia compromete a tolerância ao exercício, a qualidade de vida e a competência cognitiva. Na presente revisão encontramos 2 estudos que avaliaram a correção da 
anemia, por meio da reposição de ferro e/ou de eritropoietina, e observaram melhora significativa do $D C^{29,46}$.

Outro achado importante é a relação da inflamação e das toxinas urêmicas nos DCs associados a DRC $39,64,65,82$. Wang $J$ et al propõe estudar as alterações no equilíbrio celular Treg/Th17 e dosar suas principais citocinas no sangue periférico e seu significado em pacientes com DRC e DC. Os padrões Treg e Th17 foram analisados através da citometria de fluxo que avaliou as citocinas relevantes (IL-17, IL-10 e TGF- $\beta$ ) e outros indicadores bioquímicos, incluindo proteína $C$ reativa (PCR) e IL-6 que foram determinados por ELISA. As células Treg desempenham um papel importante na manutenção da homeostase imune e na indução de tolerância imune principalmente por secreção de IL-10, IL35, TGF- $\beta$ e outros fatores anti-inflamatórios enquanto as células Th17 promovem inflamação através da secreção de IL-17, IL-23, IL-6 e outras citocinas e estão intimamente associadas a doenças autoimunes, infecção crônica e tumores. O balanço Treg/Th17 desempenha um papel fundamental na manutenção da estabilidade do estado imunológico e seu desequilíbrio no sangue periférico está associado ao comprometimento cognitivo em pacientes com $\mathrm{DRC}^{39}$.

Vinothkumar et al ${ }^{46}$ avaliando a participação do beta amiloide na DRC, descrevem que a disfunção cognitiva afeta potencialmente até $60 \%$ desses pacientes. A GSK-3 $\beta$ (beta glycogen synthase kinase 3 ) desempenha um papel fundamental na patogênese da DA e da disfunção cognitiva, 
contribuindo para a produção de $A \beta$ e a morte neuronal mediada por $A \beta$ por fosforilação da tau, induzindo hiperfosforilação em filamentos helicoidais emparelhados. No entanto, estudos demonstraram que no plasma $\mathrm{p}$-Tau 181 é mais específico para DA e disfunção cognitiva. O mesmo foi proposto por Gronewold et ${ }^{162}$ baseado nos níveis plasmáticos de $A \beta$ que foram investigados como candidatos a biomarcadores para a patologia pré-clínica da doença de Alzheimer, mas com resultados inconsistentes e como os rins estão envolvidos na eliminação de $A \beta$ no sangue, avaliou-se a possibilidade DRC comprometer a eliminação, aumentando os níveis plasmáticos do $A \beta$ agravando $O$ declínio cognitivo.

Nos diversos estudos com uso de neuroimagem foram utilizadas imagens de Ressonância Magnética encefálica (RM) em aparelhos de 1,5 e 3 teslas e protocolos prédeterminados objetivando o estudo do volume encefálico global e focal (perda de volume cortical global e lobares), volume e intensidade das lesões da substância branca, presença de eventos vasculares isquêmicos e/ou hemorrágicos (inclusive microhemorragias) e correlacionálos com o DC. Chiu et $a^{14}$ descrevem volume cerebral total e volume subcortical reduzidos incluindo hipocampos, encontrados em pacientes em diálise. A análise mostrou afinamento cortical na região frontal média, occipital lateral e pré-cuneus. Além disso, a integridade da substância branca diminuída foi encontrada principalmente no trato talâmico anterior bilateral, fascículo fronto-occipital, fórceps menor e 
trato uncinado ${ }^{29}$. Em pacientes com DRC, a progressão da atrofia cerebral é mais rápida e o comprometimento cognitivo é mais comum do que em indivíduos não-DRC. Há relato do uso de Espectroscopia por RM em estudo realizado por Tryc, $A B$ et al em 2011, que conclui que em DRC grave a disfunção cognitiva pode ocorrer mesmo sem sinais clínicos de encefalopatia urêmica Memória e aprendizagem e déficits de atenção são as funções mais comprometidas ${ }^{82}$.

A avaliação eletroencefalográfica (EEG) foi utilizada por Lizio et a ${ }^{59}$ comparando o EEG de pacientes com CCL em DA com pacientes com CCL em DRC, encontraram resultados que sugerem que a DA precoce afeta a sincronização neural cortical em frequências alfa (sustentam a excitação cerebral na vigília e no repouso). Em contraste, a DRC pode afetar principalmente a sincronização neural cortical nas frequências delta. Futuros estudos prospectivos de validação cruzada terão que testar esses candidatos a marcadores EEG para aplicações clínicas e descoberta de medicamentos. Chao et $a l^{43}$ utilizando o EEG quantitativo objetivando examinar a diferenças nas atividades corticais de pacientes com DRC avançada, com base em seus níveis de fragilidade e implicações clínicas, concluiu que pacientes sem fragilidade e com fragilidade leve tiveram poder delta significantemente maior no EEG quantitativo do que aqueles com fragilidade moderada a grave.

Estudos experimentais de Lv et $a^{35} \mathrm{em}$ ratos refere que hipocampo desempenha um papel importante na aprendizagem espacial e na memória. A grelina, um peptídeo 
cérebro-intestinal, participa das funções mnesticas hipocampais por meio de seu receptor de secretagogo do hormônio do crescimento (GHS-R) distribuído no hipocampo. Este estudo investigou se havia uma correlação entre as alterações do sistema grelina no hipocampo e o comprometimento cognitivo espacial causado pela DRC e conclui que as alterações causadas pela DRC no sistema grelina do hipocampo podem estar correlacionadas com o comprometimento da função cognitiva. Em um segundo estudo realizado por Nakagawa et $a^{31}$ baseados em estudos epidemiológicos que sugeriam que a DRC seria um fator de risco significativo no desenvolvimento de declínio cognitivo, porém com fisiopatologia ainda desconhecida. O objetivo foi avaliar o potencial impacto da DRC no comprometimento cognitivo na doença de Alzheimer (DA), com foco na angiotensina II. A DRC foi induzida em camundongos 5XFAD, um camundongo modelo $A D$ e camundongos do tipo selvagem, alimentandos uma dieta contendo adenina e o efeito na função cognitiva foi comparado entre as duas linhagens. Não houve diferença significante em relação à gravidade da DRC induzida por adenina entre as cepas. Em camundongos 5XFAD, o grupo com DRC exibiu comprometimento cognitivo significativo, fato este não observado no grupo controle (grupo controle alimentado com dieta). Por outro lado, em camundongos do tipo selvagem, nem o grupo com DRC nem o grupo controle apresentaram comprometimento cognitivo. Assim, a própria DRC parece acelerar o comprometimento cognitivo em camundongos 
modelos de DA. E, para avaliar o papel da angiotensina II nestes casos foi utilizado o olmesartan, um bloqueador do receptor da angiotensina II, em camundongos 5XFAD com DRC. Animais 5XFAD tratados com olmesartan apresentaram melhoras significativas no comprometimento da aprendizagem espacial e da função de memória induzida pela DRC. O tratamento com olmesartan melhorou significativamente a ruptura da barreira hematoencefálica (BBB) induzida pela DRC e reduziu o estresse oxidativo no hipocampo para níveis semelhantes ao grupo controle (animais do 5XFAD alimentados com dieta padrão). Portanto, a melhora do comprometimento cognitivo induzido pela DRC em camundongos 5XFAD pelo olmesartan parece ser mediada pela supressão da interrupção da BBB ou do estresse oxidativo. Em conclusão, obtivemos evidências sugerindo que a própria DRC acelera o comprometimento cognitivo em camundongos com DA, através da angiotensina II. Assim, este trabalho fornece uma nova possível visão do mecanismo subjacente do link entre DRC e DA.

Em vários estudos associa-se a presença dos DC com impactos altamente negativos no tratamento e aderência terapêutica dos pacientes com DRC. Harhay et al/56 referem que o comprometimento cognitivo impactará no planejamento terapêutico e na preparação do paciente para o estágio terminal da doença renal, repercutindo na modalidade da terapia renal substitutiva e na lista de espera para o transplante, sendo o mesmo relatado por Wang et $a^{39}$. 


\section{Limitações dos estudos avaliados e relevância clínica}

da revisão

Importante ressaltar alguns pontos, o primeiro é diagnóstico tardio do CCL em pacientes com DRC. O segundo é a falta de padronização dos testes utilizados para investigação da função cognitiva nesta população que, como observado na revisão, deve ir para além do padronizado pelas diretrizes das sociedades de neurologia. Salientamos que, o entendimento destas limitações nos leva a reflexão sobre a importância da avaliação adequada e precoce do CCL com condutas apropriadas. Além disso, existe a necessidade de estudos de intervenção, principalmente tendo como foco as comorbidades apresentadas por estes pacientes e que são raramente mencionadas nos estudos avaliados, como doenças cardiovasculares e diabetes mellitus que tem impacto direto na função cognitiva. As alterações especificas inerentes a DRC ainda carecem de maior entendimento.

\section{CONCLUSÕES}

O CCL e a demência são frequentes em pacientes com DRC ainda na pré-diálise e a etiologia permanece em discussão. A maioria dos estudos realizados para avaliação do CCL na DRC é derivada de dados secundários de grandes coortes ou estudos transversais.

Os instrumentos clínicos mais utilizados são o MEEM e o MoCA e para avaliar do ponto de vista da neuroimagem a RM. Poucos estudos avaliam o padrão de resposta inflamatória e as toxinas urêmicas. 
Em nossa revisão apenas um estudo experimental avaliou intervenção terapêutica e este, relacionou uso de BRA com demência. Por outro lado, estudos em humanos mostram melhora do CCL por meio da utilização de EPO para correção da anemia.

Os mecanismos da DC na DRC são diversos, nem sempre correlacionados, existindo uma grande lacuna no melhor entendimento da associação fisiopatológica destas duas importantes morbidades.

\section{AGRADECIMENTOS}

Ao Programa de Pós Graduação em Saúde da Universidade Federal de Juiz de Fora (PPGS-UFJF).

\section{REFERÊNCIAS}

1.Teixeira H. Ciências da aprendizagen - Teorias e conceitos básicos O que é cognição? (endereço na internet). (atualizado em 2015; acessado em 10/10/2020). Disponível em: http://www.helioteixeira.org/ciencias-da-aprendizagem/teorias-econceitos-chava-o-que-e-cognicao/

2. Rosano A, Botto LD, Botting B, Mastroiacovo P. Infant mortality and congenital anomalies from 1950 to 1994: an international perspective. J Epidemiol Community Health 2000;54:660-6. https://doi.org/10.1136/jech.54.9.660

3.Verity $\mathrm{C}$, Firth $\mathrm{H}$, French-Constant $\mathrm{C}$. Congenital abnormalities of the central nervous system. J Neurol Neurosurg Psychiatry 2003;74(Suppl I):i3-8. https://doi.org/10.1136/jnnp.74.suppl 1.i3

4.Barros ML, Fernandes DA, Melo EV, Porto RLS, Maia MCA, Godinho AS, et al. Malformações do sistema nervoso central e malformações associadas diagnosticadas pela ultrassonografia obstétrica. Radiol Bras 2012;45:309-14. 39842012000600005

https://doi.org/10.1590/S01005.Gallucci NJ, Tamelini MG, Forlenza OV. Diagnóstico diferencial das demências. Rev Psiq Clín 2005;32:119-30. https://doi.org/10.1590/S0101-60832005000300004 
6. Radanovic M, Stella F, Forlenza O. Comprometimento cognitivo leve Mild cognitive impairment. Rev Med 2015;94:162-8. doi.org/10.11606/issn.1679-9836.v94i3p162-168

7.Sociedade Brasileira de Diabetes. Diretrizes Sociedade Brasileira de Diabetes 2019/2020. São Paulo: Clannad Ed Científica. https://www.diabetes.org.br/profissionais/images/DIRETRIZES-

COMPLETA-2019-2020.pdf

8.Department of Economic and Social Affairs. World Population Ageing 2017 - Highlights. New York: United Nations, 2017. https://www.un.org/en/development/desa/population/publications/pd f/ageing/WPA2017_Highlights.pdf

9.Instituto Brasileiro de Geografia e Estatistica - IBGE (endereço na internet). Pesquisa Nacional por Amostra de Domicílios Contínua - Pnad Contínua/maio de 2019. (acessado em 10/10/2020). Disponível em: https://www.ibge.gov.br/estatisticas/sociais/trabalho/17270-pnadcontinua.html? =\&t=0-que-e

10.Lopes MA, Bottino CMC. Prevalência de demência em diversas regiões do mundo- Análise dos estudos epidemiológicos de 1994 a 2000. Arq Neuropsiquiatr 2002;60:61-9. doi.org/10.1590/50004$\underline{282 X 2002000100012}$

11.Scazufca M, Cerqueira ATAR, Menezes PR, Prince M, Vallada HP, Miyazaki MCOS, et al. Investigações epidemiológicas sobre demência nos países em desenvolvimento. Rev Saúde Pública 2002;36:773-8. https://doi.org/10.1590/S0034-89102002000700018

12. Caramelli $P$, Carvalho VA. Doença de Alzheimer. In: Teixeira AL, Caramelli P. Neurologia Cognitiva e do Comportamento. Rio de Janeiro: Revinter, 2012, p.246-59.

13. Engelhardt E, Moreira DM. Demência Vascular. In: Teixeira AL, Caramelli P. Neurologia Cognitiva e do Comportamento Rio de Janeiro: Revinter, 2012, p.297-310.

14.Bello AK, Levin A, Lunney M, Osman MA, Ye F, Ashuntantang G, et al. Global Kidney Health Atlas: A report by the International Society of Nephrology on the Global Burden of End-stage Kidney Disease and Capacity for Kidney Replacement Therapy and Conservative Care across World Countries and Regions (endereço na internet). International Society of Nephrology, Brussels (acessado em 10/10/2020. Disponível em: www.theisn.org/global-atlas

15.Vos T, Lim SS, Abbafati C, Abbas KM, Abbasi M, Abbasifard M, et al. Global burden of 369 diseases and injuries in 204 countries and territories, 1990-2019: a systematic analysis for the Global Burden of Disease Study 2019. Lancet 2020;396:1204-22. https://doi.org/10.1016/s0140-6736(20)30925-9

16.Schmidt MI, Duncan BB, Silva GA, Menezes AM, Monteiro CA, Barreto SM, et al. Chronic non-communicable diseases in Brazil: burden and current challenges. Lancet 2011;377:1949-61. https://doi.org/10.1016/s01406736(11)60135-9

17.Kurella TM, Yaffe K, Hsu C, Yang J, Sozio S, Fischer M, et al. Cognitive Impairment and Progression of CKD. Am J Kidney Dis 2016;68:77-83. https://doi.org/10.1053/j.ajkd.2016.01.026 
18. Levin A, Stevens PE, Bilous RW, Coresh J, Francisco ALM, De Jong $\mathrm{PE}$, et al. Kidney Disease: Improving Global Outcomes (KDIGO) CKD Work Group. KDIGO 2012 Clinical Practice Guideline for the Evaluation and Management of Chronic Kidney Disease. Kid Int Suppl 2013;3:1150. https://doi.org/10.1038/kisup. 2012.73

19. Etgen T, Chonchol M, Förstl H, Sander D. Chronic Kidney Disease and Cognitive Impairment: A Systematic Review and Meta-Analysis. Am J Nephrol 2012;35:474-82. https://doi.org/10.1159/000338135

20.Donald M, Kahlon BK, Beanlands $H$, Straus $S$, Ronksley $P$, Herrington $G$, et al. Self-management interventions for adults with chronic kidney disease: a scoping review. BMJ Open 2018;8:e019814. https://doi.org/10.1136/ bmjopen-2017-019814

21.Blatt B, Tsushima WT. A Psychological Survey of Uremic Patients Being Considered for the Chronic Hemodialysis Program: Intellectual and Emotional Patterns in Uremic Patients. Nephron 1966;3:206-8. https://doi.org/10.1159/000179535

22.Tyler HR. Neurologic disorders in renal failure. Am J Med 1968;44:734-46. https://doi.org/10.1016/0002-9343(68)90255-6

23. Heilman KM, Moyer RS, Melendex F, Schwartz HD, Miller BD. A Memory Defect in Uremic Encephalopathy. J Neurol Sci 1975;26:2459. https://doi.org/10.1016/0022-510x(75)90036-2

24.Stewart RS, Stewart RM. Neuropsychiatric aspects of chronic renal disease. Psychosomatics

$1979 ; 20: 524-31$.

https://doi.org/10.1016/s0033-3182(79)70775-4

25. Brouns R, De Deyn PP. Neurological complications in renal failure: a review. Cli Neurol Neurosurg 2004;107:1-16. https://doi.org/10.1016/j.clineuro.2004.07.012

26.Burn DJ, Bates D. Neurology and the kidney. J Neurol Neurosurg Psychiatr 1998;65:810-82. https://doi.org/10.1136/jnnp.65.6.810

27. Kuriyama N, Mizuno T, Ohshima Y, Yamada K, Ozaki E, Shigeta M, et al. Intracranial deep white matter lesions (DWLs) are associated with chronic kidney disease (CKD) and cognitive impairment: A 5-year follow-up magnetic resonance imaging (MRI) study. Arc Gerontol Geriatr 2013;56:55-60.

https://doi.org/10.1016/j.archger.2011.11.009

28. Tsuruya K, Yoshida H, Haruyama N, Fujisaki K, Hirakata H, Kitazono T. Clinical Significance of Fronto-Temporal Gray Matter Atrophy in Executive Dysfunction in Patients with Chronic Kidney Disease: The VCOHP Study. PLoS One 2015;10:e0143706. https://doi.org/10.1371/journal.pone.0143706

29. Tsuruya $\mathrm{K}$, Yoshida $\mathrm{H}$. Brain Atrophy and Cognitive Impairment in Chronic Kidney Disease. Rec Adv Dial Ther Japan 2018;196:27-36. https://doi.org/10.1159/000485694

30.Shen Z, Ruan Q, Yu Z, Sun Z. Chronic kidney disease-related physical frailty and cognitive impairment: a systemic review. Geriatr Gerontol Inter 2016;17:529-44. https://doi.org/10.1111/ggi.12758

31. Nakagawa T, Hasegawa Y, Uekawa K, Kim-Mitsuyama S. Chronic kidney disease accelerates cognitive impairment in a mouse model of 
Alzheimer's disease, through angiotensin II. Exp Gerontol 2017;87:108-12. https://doi.org/10.1016/j.exger.2016.11.012

32. Yao H, Araki Y, Takashima Y, Uchino A, Yuzuriha T, Hashimoto M. Chronic Kidney Disease and Subclinical Brain Infarction Increase the Risk of Vascular Cognitive Impairment: The Sefuri Study. J Stroke Cerebrovascul Dis 2017;26:420-4.

https://doi.org/10.1016/j.jstrokecerebrovasdis.2016.10.002

33. Otobe Y, Hiraki K, Hotta C, Nishizawa H, Izawa KP, Taki Y, et al. Mild cognitive impairment in older adults with pre-dialysis patients with chronic kidney disease: Prevalence and association with physical function. Nephrology 2019;24:50-5.

https://doi.org/10.1111/nep.13173

34. Wang H, Fang C, Cai L, Dong B, Deng J. Chronic kidney disease and cognitive impairment among the very old in China. Aging Clin Exp Res 2015;28:475-82. https://doi.org/10.1007/s40520-015-0433-1

35.Lv Z, Gao J, Wang L, Chen Z, Yuan H, Ma X, et al. Uremia-caused changes of ghrelin system in hippocampus may be associated with impaired cognitive function of hippocampus. Inter Urol Nephrol 2016;48:807-15. https://doi.org/10.1007/s11255-016-1228-9

36.Cheng Z, Lin J, Qian Q. Role of Vitamin D in Cognitive Function in Chronic Kidney Disease. Nutrients 2016;8:291. https://doi.org/10.3390/nu8050291

37.Pi HC, Xu YF, Xu R, Yang ZK, Qu Z, Chen YQ, et al. Cognitive Impairment and Structural Neuroimaging Abnormalities Among Patients with Chronic Kidney Disease. Kidney Blood Pres Res 2016;41:986-96. https://doi.org/10.1159/000452603

38. Bai K, Pan Y, Lu F, Zhao Y, Wang J, Zhang L. Kidney function and cognitive decline in an oldest-old Chinese population. Clin Intervent Aging 2017;12:1049-54. https://doi.org/10.2147/cia.s134205

39.Wang J, Li XB, Huang P, Huang MY, Gu XJ. Change of Peripheral Blood Treg/Thl7 in Cognitive Impairment with Chronic Renal Failure Patients. Cell Physiol Biochemist 2018;45:281-90. https://doi.org/10.1159/000486

40. Chen YC, Weng SC, Liu JS, Chuang HL, Hsu CC, Tarng DC. Severe Decline of Estimated Glomerular Filtration Rate Associates with Progressive Cognitive Deterioration in the Elderly: A Community-Based Cohort Study. Sci Rep 2017;7:42690. https://doi.org/10.1038/srep42690

41.Chang CY, Lin CC, Tsai CF, Yang WC, Wang SJ, Lin FH, et al. Cognitive impairment, and hippocampal atrophy in chronic kidney disease. Acta Neurol Scan 2017;136:477-85. https://doi.org/10.1111/ane.12753

42. Chen $\mathrm{CH}$, Chen YF, Chiu MJ, Chen TF, Tsai PH, Chen JH, et al. Effect of Kidney Dysfunction on Cerebral Cortical Thinning in Elderly Population. Sci Rep 2017;7:2337. 1_https://doi.org/0.1038/s41598017-02537-y

43. Chao CT, Lai HJ, Tsai HB, Yang SY, Huang JW. Frail phenotype is associated with distinct quantitative electroencephalographic findings 
among end-stage renal disease patients: an observational study. BMC Geriatr 2017;17:277. https://doi.org/10.1186/s12877-017-0673-3

44. Chiu YL, Tsai HH, Lai Y], Tseng HY, Wu YW, Peng YS, et al. Cognitive impairment in patients with end-stage renal disease: Accelerated brain aging? J Formos Med Assoc 2019;118:867-75. https://doi.org/10.1016/j.jfma.2019.01.011

45.Vinothkumar G, Krishnakumar S, Sureshkumar, Shivashekar G, Sreedhar S, Preethikrishnan, et al. Therapeutic impact of rHuEPO on abnormal platelet APP, BACE 1, presenilin 1, ADAM 10 and A $\beta$ expressions in chronic kidney disease patients with cognitive dysfunction like Alzheimer's disease: A pilot study. Biomed Pharmacother 2018;104:211-22.

https://doi.org/10.1016/j.biopha.2018.05.028

46. Vinothkumar G, Krishnakumar S, Riya, Venkataraman P. Correlation between abnormal GSK3 $\beta$, $\beta$ Amyloid, total Tau, p-Tau 181 levels and neuropsychological assessment total scores in CKD patients with cognitive dysfunction: Impact of rHuEPO therapy. J Clin Neurosci 2019;69:38-42. https://doi.org/10.1016/j.jocn.2019.08.073

47.Yeh YC, Huang MF, Hwang SJ, Tsai JC, Liu TL, Hsiao SM, et al. Association of homocysteine level and vascular burden and cognitive function in middle-aged and older adults with chronic kidney disease. Inter J Geriatr Psychiatr 2015;31:723-30. https://doi.org/10.1002/gps.4383

48. Kittiskulnam P, Sheshadri A, Johansen KL. Consequences of CKD on Functioning. Sem Nephrol 2016;36:305-18. https://doi.org/10.1016/j.semnephrol.2016.05.007

49.Zammit AR, Katz MJ, Bitzer M, Lipton RB. Cognitive Impairment and Dementia in Older Adults With Chronic Kidney Disease: A Review. Alzheimer Dis Assoc Disord 2016;30:357-66. doi.org/10.1097/WAD.0000000000000178

50. Jasinski MJ, Lumley MA, Soman S, Yee J, Ketterer MW. Indicators of Cognitive Impairment From a Medical Record Review: Correlations With Early (30-d) Readmissions Among Hospitalized Patients in a Nephrology Unit. Psychosomatics 2017;58:173-9. https://doi.org/10.1016/j.psym.2016.10.005

51.Torres RV, Elias MF, Seliger S, Davey A, Robbins MA. Risk for cognitive impairment across 22 measures of cognitive ability in earlystage chronic kidney disease. Nephrol Dial Transpl 2016;32:299-306. https://doi.org/10.1093/ndt/gfw005

52. Bronas UG, Puzantian $H$, Hannan M. Cognitive Impairment in Chronic Kidney Disease: Vascular Milieu and the Potential Therapeutic Role of Exercise. BioMed Res Inter 2017;ID2726369. https://doi.org/10.1155/2017/2726369

53. Freedman BI, Sink KM, Hugenschmidt CE, Hughes TM, Williamson JD, Whitlow CT, et al. Associations of Early Kidney Disease With Brain Magnetic Resonance Imaging and Cognitive Function in African Americans With Type 2 Diabetes Mellitus. Am J Kid Dis 2017;70:62737. https://doi.org/10.1053/j.ajkd.2017.05.006 
54. Materson BJ, Garcia-Estrada M, Degraff SB, Preston RA. Prehypertension is real and can be associated with target organ damage. J Am Soc Hypert 2017;11:704-8. https://doi.org/10.1016/j.jash.2017.09.005

55.Burns CM, Knopman DS, Tupper DE, Davey CS, Slinin YM, Lakshminarayan $\mathrm{K}$, et al. Prevalence and Risk of Severe Cognitive Impairment in Advanced Chronic Kidney Disease. J Gerontol A Biol Sci Med Sci 2018;73:393-9. https://doi.org/10.1093/gerona/glx241

56. Harhay MN, Xie D, Zhang X, Hsu C, Vittinghoff E, Go AS, et al. Cognitive Impairment in Non-Dialysis-Dependent CKD and the Transition to Dialysis: Findings From the Chronic Renal Insufficiency Cohort (CRIC) Study. Am J Kid Dis 2018;72:499-508. https://doi.org/10.1053/j.ajkd.2018.02.361

57. Coppolino G, Bolignano D, Gareri P, Ruberto C, Andreucci M, Ruotolo $\mathrm{G}$, et al. Kidney function and cognitive decline in frail elderly: two faces of the same coin? Inter Urol Nephrol 2018;50:1505-10. https://doi.org/10.1007/s11255-018-1900-3

58. Guenzani D, Buoli M, Carnevali GS, Serati M, Messa P, Vettoretti S. Is there an association between severity of illness and psychiatric symptoms in patients with chronic renal failure? Psychol Health Med 2018;23:970-9. https://doi.org/10.1080/13548506.2018.1426868

59. Lizio R, Babiloni C, Del Percio C, Losurdo A, Vernò L, De Tommaso $M$, et al. Different Abnormalities of Cortical Neural Synchronization Mechanisms in Patients with Mild Cognitive Impairment due to Alzheimer's and Chronic Kidney Diseases: An EEG Study. J Alzheimers Dis 2018;65:897-915. https://doi.org/10.3233/JAD-180245

60. Kallenberg MH, Kleinveld HA, Dekker FW, van Munster BC, Rabelink $\mathrm{TJ}$, van Buren $\mathrm{M}$, et al. Functional and Cognitive Impairment, Frailty, and Adverse Health Outcomes in Older Patients Reaching ESRD - A Systematic Review. Clin J Am Soc Nephrol 2016;11:1624-39. https://doi.org/10.2215/cjn.13611215

61.Berkhout-Byrne N, Kallenberg $\mathrm{MH}$, Gaasbeek A, Rabelink TJ, Hammer S, van Buchem MA, et al. The cognitive decline in older patients with end stage renal disease (COPE) study - rationale and design. Curr Med Res Op 2017;33:2057-64. https://doi.org/10.1080/03007995.2017.1341404

62. Gronewold J, Todica O, Seidel UK, Volsek M, Kribben A, Bruck H, et al. Cognitive Performance Is Highly Stable over a 2-Year-Follow- Up in Chronic Kidney Disease Patients in a Dedicated Medical Environment. PLoS One 2017;11:e0166530.

https://doi.org/10.1371/journal.pone.0166530

63. König M, Gollasch M, Spira D, Buchmann N, Hopfenmüller W, Steinhagen-Thiessen $\mathrm{E}$, et al. Mild-to-Moderate Chronic Kidney Disease and Geriatric Outcomes: Analysis of Cross-Sectional Data from the Berlin Aging Study II. Gerontology 2017;64:118-26. https://doi.org/10.1159/000484140

64.Puy L, Bugnicourt JM, Liabeuf S, Desjardins L, Roussel M, Diouf M, et al. Cognitive Impairments and Dysexecutive Behavioral Disorders in 
Chronic Kidney Disease. J Neuropsychiatr Clin Neurosci 2018;30:3107. https://doi.org/10.1176/appi. neuropsych.18030047

65. Karasavvidou $D$, Kalaitzidis $R$, Elisaf $M$. Arterial damage and cognitive impairment in patients with chronic kidney disease. J Clin Hyperten 2018;20:1643. https://doi.org/10.1111/jch.13402 66. Paraizo MA, Almeida ALM, Pires LA, Abrita RSA, Crivellari MHT, Pereira BS, et al. Montreal Cognitive Assessment (MoCA) screening mild cognitive impairment in patients with chronic kidney disease (CKD) pre-dialysis. J Bras Nefrol 2016;38:31-41. https://doi.org/10.5935/0101-2800.20160006

67. Passos VMA, Ladeira RM, Souza CCV, Santos IS,Barreto SM. First stages chronic kidney disease have mild effects on cognitive performance. Results of a 15,105 Brazilian adult baseline cohort. Braz J Nephrol 2018;40:18-25. https://doi.org/10.1590/1678-4685-jbn$\underline{3889}$

68. Franco AO, Starosta RT, Roriz-Cruz M. The specific impact of uremic toxins upon cognitive domains: a review. Braz J Nephrol 2018;41:10311. https://doi.org/10.1590/2175-8239-jbn-2018-0033

69. Amatneeks TM, Hamdan AC. Sensitivity and specificity of the Brazilian version of the Montreal Cognitive Assessment - Basic (MoCA$B$ ) in chronic kidney disease. Trends Psychiatr Psychother 2019;41:327-33. doi.org/10.1590/2237-6089-2018-0085

70.Chaiben VBO, Silveira TB, Guedes MH, Fernandes JPA, Ferreira JHF, Beltrão J, et al. Cognition and renal function: findings from a Brazilian population. Braz J Nephrol 2019;41:200-7. https://doi.org/10.1590/2175-8239-jbn-2018-0067

71. Foster R, Walker S, Brar R, Hiebert B, Komenda P, Rigatto C, et al. Cognitive Impairment in Advanced Chronic Kidney Disease: The Canadian Frailty Observation and Interventions Trial. Am J Nephrol 2016;44:473-80. https://doi.org/10.1159/000450837

72. Assayag E, Eldor R, Korczyn AD, Kliper E, Shenhar-Tsarfaty S, Tene $O$, et al. Type 2 Diabetes Mellitus and Impaired Renal Function Are Associated With Brain Alterations and Poststroke Cognitive Decline. Stroke 2017;48:2368-74.

https://doi.org/10.1161/strokeaha.117.017709

73.Szerlip HM, Edwards ML, Williams BJ, Johnson LA, Vintimilla RM, O'Bryant SE. Association Between Cognitive Impairment and Chronic Kidney Disease in Mexican Americans. J Am Geriatr Soc 2015;63:20238. https://doi.org/10.1111/jgs. 13665

74. Folstein MF, Folstein SE, McHugh PR. Mini-Mental State: a practical method for grading the cognitive state for the clinician. J Psychiatr Res 1975;12:189-98. https://doi.org/10.1016/0022-3956(75)90026-6

75. Bertolucci PHF, Brucki SMD, Campacci AR, Juliano Y. O mini-exame do estado mental em uma população geral: impacto da escolaridade. Arq Neuropsiquiar 1994;52:1-7. https://doi.org/10.1590/S0004282X1994000100001

76.Teng EL, Chui HC. The Modified Mini-Mental State (3MS) Examination. J Clin Psychiatr 1987;48:314-8. 
77.Teng EL, Chui HC, Gong A. Comparisons between the Mini-Mental State Exam (MMSE) and its modified version - the 3MS test. In: Hasegawa K, Homma A (Ed.). Psychogeriatrics: biomedical and social advances. Amsterdam: Excerpta Medica, 1990, p.189-92.

78. Nasreddine ZS, Phillips NA, Bedirian V, Charbonneau S, Whitehead V, Collin I, et al. The Montreal Cognitive Assessment, MoCA: A Brief Screening Tool For Mild Cognitive Impairment. J Am Geriatric Soc 2005;53:695-9. https://doi.org/10.1111/j.1532-5415.2005.53221.x 79. European Commission, Directorate General Research and Innovation. Functional Magnetic Resonance Imaging - Understanding the technique and addressing its ethical concerns with a future perspective. (Acessado em 10/10/2020). 2013. Disponível em: https://www.researchgate.net/publication/303820836 Functional Ma gnetic Resonance Imaging Understanding the technique and addr essing its ethical concerns with a future perspective httpeceuropa euresearchparticipantsdatarefh2020otherhiethics-guide-

fmri e/link/5755ec1d08ae10c72b66ec09/download

80.Sakamoto SC. Estudo da variabilidade do efeito BOLD em ressonância magnética funcional em sistema de 3 teslas (Tese). São Paulo: Universidade de São Paulo, 2016. https://doi.org/10.11606/T.5.2017.tde-03052017-144019

81. Moodalbail DG, Reiser KA, Detre JA, Schultz RT, Herrington JD, Davatzikos $C$, et al. Systematic Review of Structural and Functional Neuroimaging Findings in Children and Adults with CKD. Clin J Am Soc Nephrol 2013;8:1429-48. https://doi.org/10.2215/CJN.11601112

82. Tryc AB, Alwan G, Bokemeyer M, Goldbecker A, Hecker H, Haubitz $M$, et al. Cerebral metabolic alterations and cognitive dysfunction in chronic kidney disease. Nephrol Dia Transplant 2011;26:2635-41. https://doi.org/10.1093/ndt/gfa729 